\title{
Arqueología e historia en Aldea Beleiro (sudoeste de Chubut). Antiguos y nuevos habitantes de la cueva Casa de Piedra de Roselló y sus alrededores
}

Analía Castro Esnal" y M. Laura Casanueva ${ }^{* *}$
Recibido: 13 de marzo de 2017

Aceptado: 5 de septiembre de 2017

\section{Resumen}

Desde el año 2010 se realizan tareas de investigación arqueológica en Aldea Beleiro, sudoeste de Chubut, con la finalidad de recuperar la historia del poblamiento de la provincia, desde sus comienzos hasta la llegada de los inmigrantes en el siglo XIX y el surgimiento de la población criolla. Desde la Arqueología -analizando tanto la cultura material como documentos históricos y entrevistas- y con un enfoque regional, se busca armar el rompecabezas histórico del área. En este sentido, uno de los ejes de esta investigación es la interacción de los distintos grupos sociales y la vida cotidiana transcurrida en sus espacios de habitación. En este trabajo se presentan los resultados de los estudios llevados a cabo en la cueva Casa de Piedra de Roselló y los relevamientos realizados en asentamientos de familias indígenas de momentos históricos en Aldea Beleiro, demostrando así una continua ocupación de la región desde hace al menos 9000 años.

\section{Archeology and History at Aldea Beleiro (Southweast of Chubut): Old and new inhabitants of Casa de Piedra de Roselló Cave and its environs}

Palabras clave

Patagonia Poblamiento Arqueología Contacto cultural

\begin{abstract}
Since 2010, archaeological research has been carried out at Aldea Beleiro, Southwestern Chubut, aimed at recovering the history of the peopling of the province from its origins to the arrival of immigrants during the 19th Century, leading to the subsequent emergence of a creole population. Using archaeology, including material culture as well as historical documents and interviews, allied to a regional approach, we seek to rebuild the history of the area. In this sense, a major focus of this research is the interaction between different social groups and daily life spent in their areas of habitation. To this effect, this paper will present the results of research carried out at the Casa de Piedra de Roselló Cave, and of surveys undertaken at indigenous family settlements for the historical period at Aldea Beleiro. These demonstrate a continuous occupation of the region since at least, 9,000 years ago.
\end{abstract}

\section{Keywords}

Patagonia Peopling Archeology Cultural contact

\footnotetext{
* Instituto Nacional de Antropología y Pensamiento Latinoamericano (INAPL) - CONICET. 3 de febrero 1378 (CP C1426BJN), Ciudad Autónoma de Buenos Aires, Argentina. E-mail: analiacastro@gmail.com

** Instituto Nacional de Antropología y Pensamiento Latinoamericano (INAPL). 3 de febrero 1378 (CP C1426BJN),

Ciudad Autónoma de Buenos Aires, Argentina. E-mail: mlauracasanueva@gmail.com
} 


\section{Introducción}

La versatilidad de la Patagonia se ve reflejada en la variedad de pobladores que surcaron su territorio desde hace miles de años hasta el presente. Mediante los proyectos UBACYT F219 y CONICET (PIP 084; PIP 023), dirigidos por la Dra. Cecilia Pérez de Micou, se busca recuperar la historia del poblamiento de la provincia de Chubut desde sus comienzos hasta la llegada de los inmigrantes en el siglo XIX, focalizando la investigación en el sudoeste de Chubut y su relación con otras áreas arqueológicas cercanas.

Las modalidades del contacto entre los indígenas de Patagonia y las distintas familias de inmigrantes fueron diversas y cambiantes a través del tiempo y en distintos espacios. Las investigaciones en curso en el sudoeste de Chubut intentan recuperar los paisajes en que estas comunidades se movieron, a partir del estudio de los espacios que efectivamente ocuparon y la evaluación de los criterios de elección de los mismos. De este modo, se busca comprender las características de la movilidad de los diferentes grupos, su vida cotidiana y las particularidades de su interacción.

En este marco, desde el año 2010, se desarrollan tareas de investigación en Aldea Beleiro, comarca ubicada en una zona ecotonal entre la estepa y el bosque. En este paraje, desde hace al menos 9000 años, han vivido poblaciones indígenas como lo indica la datación de la cueva "Casa de Piedra" de la estancia Roselló (Castro Esnal, Casanueva, Sacchi y Pérez de Micou, 2016; Castro Esnal, Pérez de Micou y Casanueva, 2017a; Pérez de Micou, Castro Esnal y Sacchi, 2013) (Figura 1). Ubicada en una zona estratégica, la variedad de sus recursos y accesibilidad hacia los bosques de la cordillera, hicieron de esta cueva un lugar de asentamiento óptimo hasta momentos históricos cuando comenzaron a llegar al área los colonos pioneros a finales del siglo XIX y principios del XX (Casanueva, 2016). En este trabajo se presentan los resultados de las investigaciones llevadas a cabo en la cueva Casa de Piedra de Roselló, principalmente en sus niveles superiores y las nuevas prospecciones realizadas en asentamientos de familias indígenas de momentos históricos.

\section{Momentos iniciales de ocupación: el sitio Casa de Piedra de Roselló}

La cueva Casa de Piedra de Roselló está ubicada aproximadamente a $5 \mathrm{~km}$ al este del límite internacional con la República de Chile, en un cañadón formado por un macizo rocoso por el que discurre el arroyo Nirihuao. Se trata de un sitio compuesto por tres abrigos ubicados a unos $12 \mathrm{~m}$ de altura sobre el mallín que forma el arroyo y a unos 100 $m$ de distancia de éste (Figura 2). La excavación de la cueva principal (CP1) ha dado como resultado la recuperación de vestigios en nueve capas estratigráficas producto de una serie de ocupaciones, la primera de ellas de al menos 9000 años AP (Tabla 1) (Castro Esnal et al., 2016, 2017a; Pérez de Micou et al., 2013).

Entre los materiales recuperados en $\mathrm{CP} 1$ se destaca por su abundancia el material lítico (Tabla 2). Se trata mayoritariamente de desechos de talla de una roca silícea disponible inmediatamente en sectores del afloramiento rocoso en el que se emplaza la cueva (Formación Carrenleufú; Dal Molin, 1998) y en menor medida lascas, instrumentos y núcleos de otras materias primas tales como diversas rocas silíceas, basalto, riolita y obsidiana (Castro Esnal et al., 2016, 2017a; Pérez de Micou et al., 2013). Entre los instrumentos hallados cabe mencionar la presencia de puntas de proyectil apedunculadas halladas en las capas 5 y 6 (Castro Esnal et al., 2016, 2017a) (Figura 2).

Los restos faunísticos se encuentran en general mal preservados, posiblemente debido a la matriz húmeda de algunos de los estratos. No obstante, se ha podido identificar 


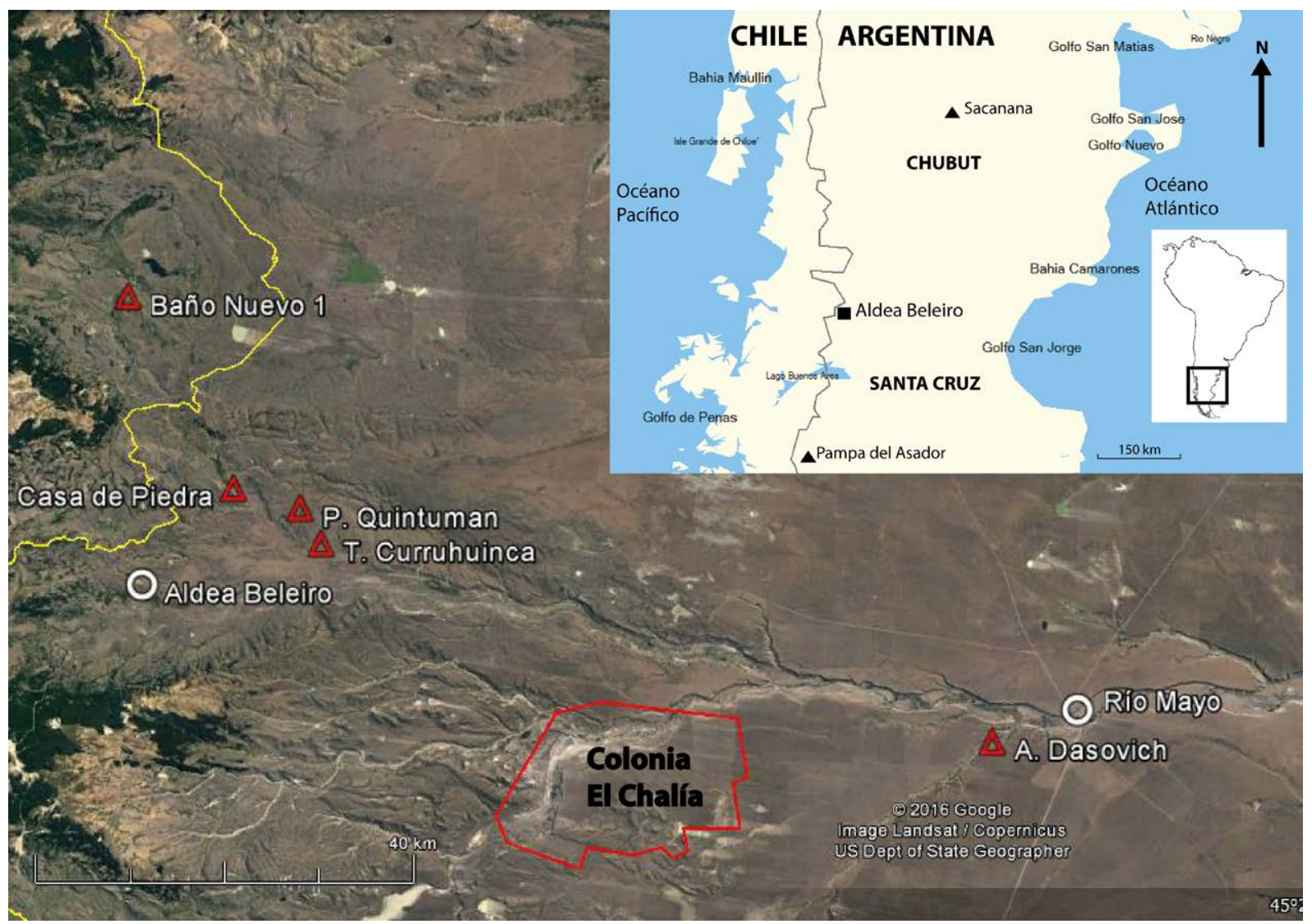

Figura 1. Mapa del área con los sitios arqueológicos mencionados en el texto.

la presencia predominante de guanaco (Lama guanicoe), algunos restos de oveja (sólo en los estratos superiores), huemul (Hippocamelus bisulcus, en capas 4 y 6) y restos de aves mediano/pequeñas.

Los otros dos abrigos (CP2 y CP3) adyacentes a $\mathrm{CP} 1$, son más pequeños y presentan pinturas rupestres (Castro Esnal et al., 2016, 2017a). En CP3 se registró la presencia de numerosos motivos pintados entre los que predominan los negativos de mano. Asimismo, se identificaron motivos abstractos, guanacos muy desvaídos y una gran cantidad de "indeterminados" debido al estado de deterioro que presentan las pinturas en general (por causas naturales y en algunos casos por la sobreimposición de inscripciones o "grafitis"). En CP2 se observaron negativos de mano aislados. En ambos abrigos se registró escaso material lítico en superficie y en CP3 hasta el momento se realizó un sondeo sin hallazgos.

Casa de Piedra de Roselló se destaca en la región por su profundidad temporal, hasta el momento comparable regionalmente con el sitio Baño Nuevo 1 en Aysén, localidad vecina de Chile (Bate, 1979; Mena, Lucero, Reyes, Trejo y Velázquez, 2000; Mena y Stafford, 2006; Reyes, Méndez, Mena y Moraga, 2012; entre otros) y con el Alero Dásovich ubicado en las proximidades de Río Mayo (Aguerre, Andrieu e Iantanos, 2017) (Figura 1). Asimismo, la posibilidad de tener una estratigrafía continua, bien conservada, que muestra ocupaciones de distintos momentos y grupos (desde cazadores recolectores hasta pobladores de tiempos históricos recientes), permite formular hipótesis relacionadas con cambios en el uso del espacio por parte de los grupos humanos a través del tiempo. En este sentido, los estudios geoquímicos realizados sobre artefactos indígenas confeccionados en obsidiana indican circuitos 


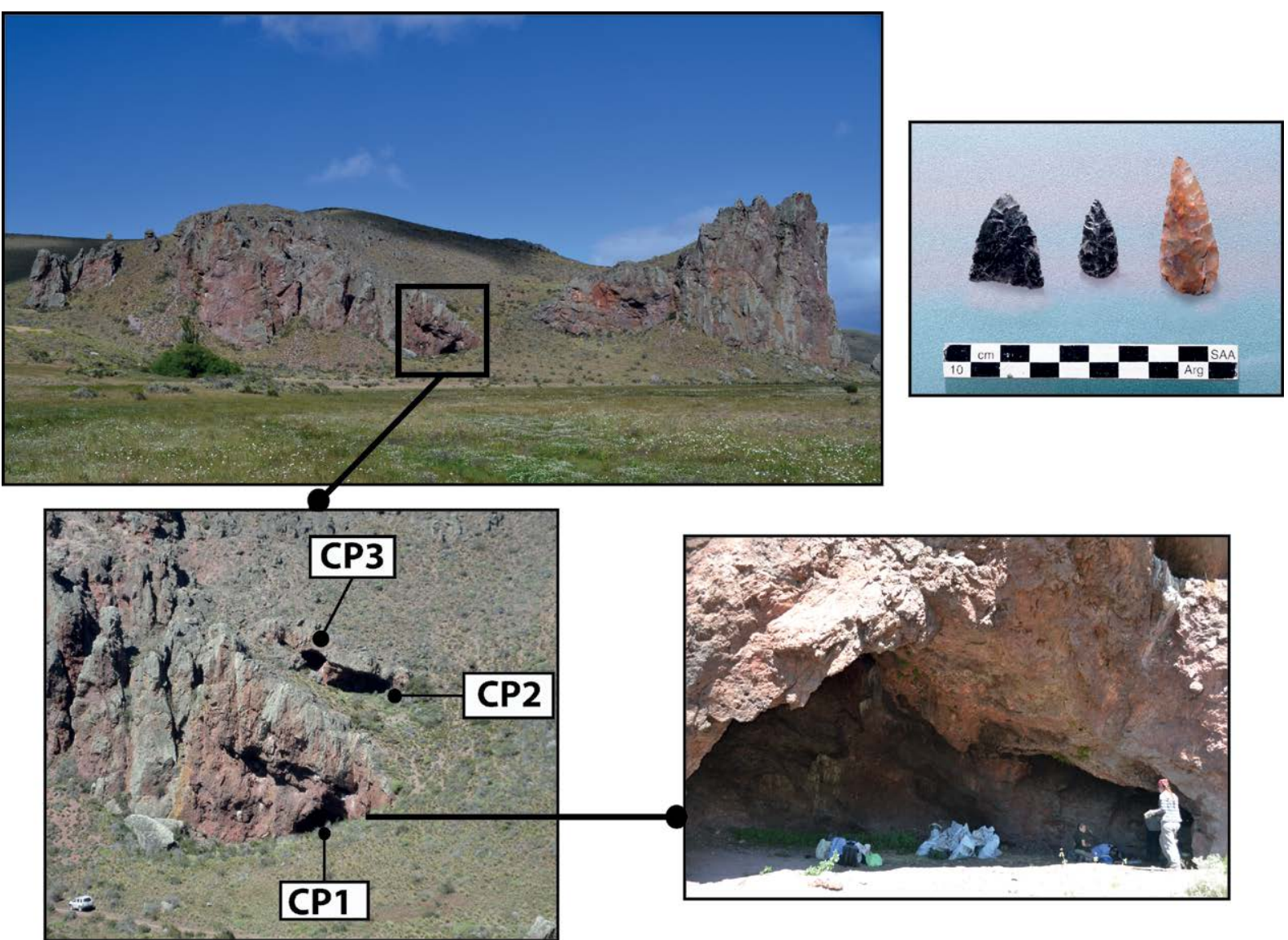

Figura 2. Vistas generales y puntas de proyectil de Casa de Piedra de Roselló.

\begin{tabular}{|l|c|c|c|c|}
\hline Muestra & Capa & Material Fechado & C14 AMS & $\begin{array}{c}\text { cal. AP (95,4 \%) } \\
\text { OxCal 4.2.Curva:ShCal13 }\end{array}$ \\
\hline AA94274 & 1 & Madera & $493 \pm 34$ & $543 / 461$ \\
AA94275 & $(2)$ & Estaca clavada & $179 \pm 34$ & Fuera de rango \\
AA103810 & 4 & Carbón vegetal & $5682 \pm 46$ & $6532 / 6305$ \\
AA103811 & 6 & Carbón vegetal & $7719 \pm 51$ & $8579 / 8397$ \\
AA105881 & 7 & Carbón vegetal & $8033 \pm 37$ & $9007 / 8658$ \\
\hline
\end{tabular}

Tabla 1. Fechados publicados hasta el momento para CP1 (Castro Esnal et al., 2016, 2017a; Pérez de Micou et al., 2013).

de movilidad y/o redes de interacción que han cambiado a lo largo del tiempo (Castro Esnal, Stern y Pérez de Micou, 2017b). La presencia de artefactos de obsidiana de Pampa del Asador (provincia de Santa Cruz, aproximadamente a $270 \mathrm{~km}$ hacia el sur de CP1) (Figura 1) pone en evidencia que desde tiempos tempranos los grupos humanos de esta zona accedieron a los recursos de amplios territorios. Además, entre los materiales líticos de sitios de superficie cercanos asignados a momentos tardíos, también se encuentra otro tipo de obsidiana de una fuente ubicada hacia el noreste: Cerro Guacho de Sacanana (370 km aproximados) (Figura 1). La obsidiana de Sacanana no fue hallada en los estratos fechados para los momentos tempranos representados en CP1, lo que estaría indicando o una ampliación en el circuito de movilidad o un cambio en las relaciones e intercambios entre grupos de cazadores recolectores (Castro Esnal et al., 2017b). 


\begin{tabular}{|c|c|c|c|c|c|c|c|c|}
\hline Capa & Desechos & $\begin{array}{c}\text { Instru- } \\
\text { mentos }\end{array}$ & Núcleos & Vidrios & Metal & $\begin{array}{c}\text { Restos } \\
\text { Fauna }\end{array}$ & Total & $\begin{array}{c}\text { Área } \\
\text { Excavada }\end{array}$ \\
\hline 0 & 33 & 3 & 2 & 11 & 6 & 10 & 65 & $2 \mathrm{~m}^{2}$ \\
1 & 631 & 11 & 3 & - & 7 & 127 & 779 & $2 \mathrm{~m}^{2}$ \\
2 & 942 & 5 & 4 & - & - & 142 & 1093 & $2 \mathrm{~m}^{2}$ \\
3 & 471 & 5 & 1 & - & - & 86 & 563 & $2 \mathrm{~m}^{2}$ \\
4 & 4420 & 109 & 8 & - & - & 1297 & 5834 & $2 \mathrm{~m}^{2}$ \\
5 & 444 & 4 & 0 & - & - & 91 & 539 & $1 \mathrm{~m}^{2}$ \\
6 & 1693 & 21 & 2 & - & - & 634 & 2350 & $1 \mathrm{~m}^{2}$ \\
7 & 191 & 1 & 0 & - & - & 8 & 200 & $1 \mathrm{~m}^{2}$ \\
8 & 24 & 1 & 0 & - & - & 0 & 25 & $1 \mathrm{~m}^{2}$ \\
\hline
\end{tabular}

Tabla 2. Material hallado en $\mathrm{CP}_{1}$ : lítico, restos faunísticos y material industrial (Clavos, alambres y pequeños vidrios) (Castro Esnal et al., 2016, 2017a).

Por otro lado, la presencia de huemul (H. bisulcus) en las capas 4 y 6 de CP1 advierte sobre el consumo de recursos provenientes del bosque, lo que señala una explotación de las áreas boscosas ubicadas hacia el oeste. Restos de huemul, en cambio, no están presentes en la ocupación tardía de CP1, lo que probablemente puede deberse a un cambio ambiental por el avance de la estepa hacia el oeste (Castro Esnal et al., 2016, 2017a).

En suma, Casa de Piedra de Roselló tiene una ubicación estratégica en cuanto al acceso a diversos recursos, reflejada en su registro arqueológico en la variedad de restos zooarqueológicos (presencia de huemul) y en el uso de materias primas alóctonas (obsidiana). Esta característica, sumada a su tamaño y orientación que la protege de los vientos del oeste, hizo de esta cueva un lugar de asentamiento óptimo hasta momentos históricos recientes, cuando a principios del siglo XX fue habitada por una familia de escasos recursos (dato surgido de las entrevistas a pobladores locales) (Pérez de Micou et al., 2013).

\section{Momentos recientes: indígenas y colonos}

El material recuperado en superficie en CP1, además del de estratigrafía (capas 0 y 1 , Tabla 2), se caracteriza por: 13 fragmentos de vidrio (tres bases de botellas, una de ellas redonda verde con push up pronunciado, marca de pontil y burbujas; un fragmento de base con cuerpo de botellón de gruesas paredes (Rock, 1981); dos golletes de frasco; un gollete de botella; cuatro fragmentos de cuerpo de botella verde; cuatro fragmentos de cuerpo de botella de cerveza); cuatro fragmentos de porrones de gres y una bisagra de metal (Figura 3). Este material es asignable a los últimos años del siglo XIX y principalmente al siglo XX. A su vez, se halló un mortero fragmentado, una bola con surco también fragmentada y escasos desechos de talla.

Los orígenes de Aldea Beleiro como poblado, se remontan a las últimas décadas del siglo XIX cuando se posicionó como sector de cruce de caminos hacia ambos lados de la cordillera, producto de la consolidación del intercambio comercial entre Chile y Argentina, siendo Sarmiento el destino principal del ganado y los productos comercializados (Maggiori, 2007; Musso, 2015; y entrevistas a pobladores locales). Entre 1910 y 1916 comenzaron a llegar los primeros inmigrantes españoles con intensión de trabajar cerca de la frontera y lograr tierras para establecerse. Rafael Beleiro y Vicente Pérez, considerados pioneros y fundadores, representaron el ideal nacional del inmigrante europeo en contraposición a la población nativa. En la versión local 


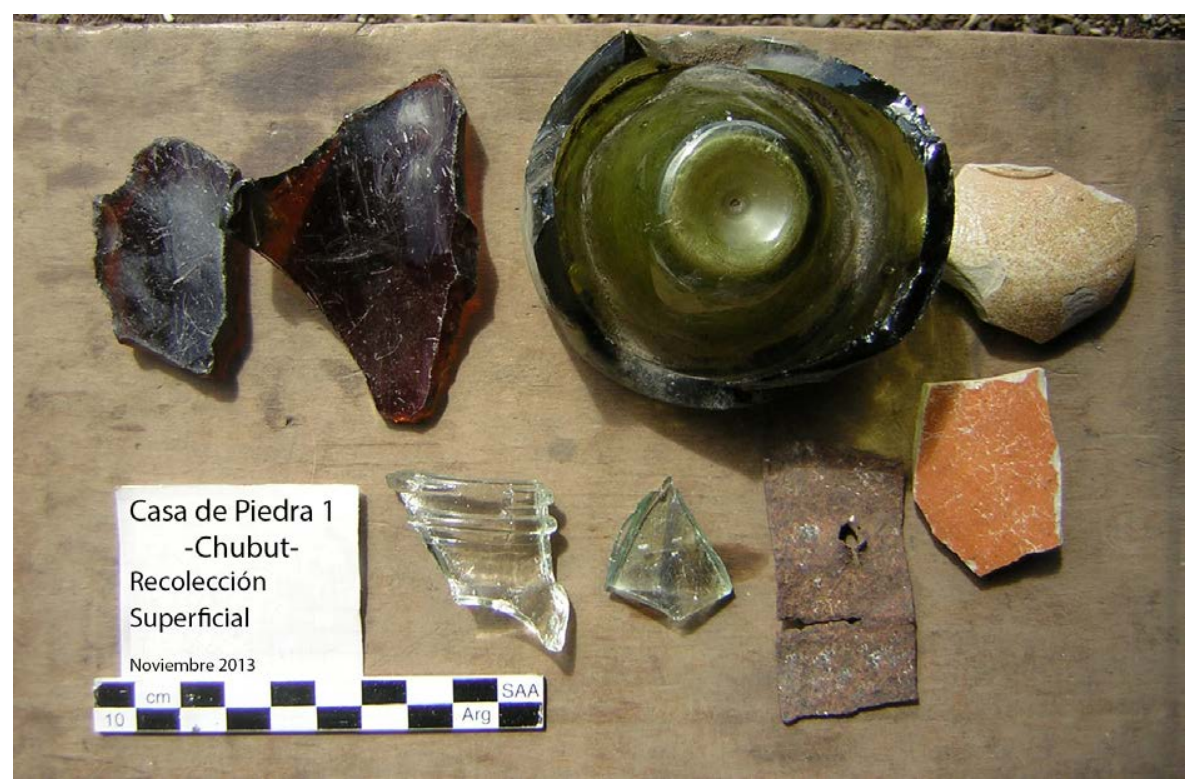

Figura 3. Parte del material "europeo/criollo" de superficie de CP1.

de este ideal, los inmigrantes europeos aparecían como los deseados, frente a los no deseados representados por chilenos y pobladores tehuelches y mapuches (Baeza, 2007). Esta política estatal, luego de la "Campaña del Desierto", tuvo como objetivo afianzar la presencia nacional en la frontera con Chile, para lo cual contó con el accionar de los inspectores de tierras, funcionales al discurso nacional de principios del siglo XX, quienes fueron los encargados de sugerir o no el otorgamiento de la propiedad de las tierras; de esta forma se fueron construyendo las diversas representaciones acerca de los habitantes de la frontera (Baeza, 2007; Del Río, 2010).

Desde la Arqueología -analizando los restos materiales, los documentos históricos y registrando la memoria de los pobladores- se intenta recuperar la micro historia local (Levi, 1993; Revel, 1995) tomando como ejes de la investigación la interacción de los distintos grupos sociales, la vida cotidiana transcurrida en sus espacios domésticos y de producción y las relaciones asimétricas que originaron el acceso diferencial a la propiedad de la tierra en espacios fronterizos. Dentro de este marco, se considera a la frontera como un vasto espacio social en el que se desarrollaron procesos históricos específicos, en donde se desenvolvieron múltiples y complejas relaciones entre la "sociedad blanca" y la indígena (Bandieri, 2005); un espacio poroso, maleable y múltiple (Quijada, 2002), en el que los indígenas han tenido un papel activo, siendo significativa su participación en la vida diaria (Ratto, 2001). En suma, la frontera es entendida como el espacio en el que se construyó una sociedad móvil, permeable y compleja (Quijada, 2002).

En definitiva, la historia reciente de Aldea Beleiro, contemporánea a los niveles superiores de CP1, fue definida decisivamente por los europeo-criollos en un contexto en el que los chilenos y principalmente los indígenas fueron quedando al margen y al servicio como puesteros, peones o jornaleros de los nuevos terratenientes ganaderos (Casanueva, 2013, 2016; Cirigliano, 2016; Nuevo Delaunay, Goñi, Jiménez y Cecuk, 2014). Tomando esta problemática como un aspecto de interés en la presente investigación, además de los hallazgos de CP1, se prospectaron dos sitios cercanos (Figura 1) de ocupación indígena de momentos recientes: Tapera Curruhuinca y Puesto Quintuman, los que fueron localizados como consecuencia de las entrevistas realizadas en la zona y la información histórica consultada (Censo de las familias Indígenas de 1927). 
El Cacique Basilio Curruhuinca y su gente llegaron a la zona, provenientes desde el área del Lago Lacar, como consecuencia de los desplazamientos y reubicaciones luego de la "Campaña del Desierto" (Habegger, 2007). Según las fuentes, se asentaron a principios del siglo XX en Alto Río Mayo, a escasos kilómetros de lo que hoy es Aldea Beleiro, en un área de buenas tierras con un importante manantial rodeado de arboleda. Con el tiempo se fueron interrelacionando con los miembros de la cercana Colonia Tehuelche del Chalía de Manuel Quilchamal, creada en 1916, ubicada al este de Aldea Beleiro a una distancia aproximada de $45 \mathrm{~km}$ (Castro Esnal, 2014; Castro Esnal, Sacchi y Pérez de Micou, 2011; Pérez de Micou, Sacchi, Castro Esnal y Funes, 2009; Pinotti, 2001, 2004; Sacchi, 2012). Sin embargo, avanzado el siglo XX, los colonos los fueron desplazando hacia áreas menos productivas a través de engaños (según surge de las entrevistas realizadas) (Castro Esnal et al., 2016). De acuerdo con el censo consultado, para 1927 Curruhuinca y su gente estaban asentados en viviendas de adobe y zinc; contaban a su vez, con galpones y corrales, haciendas y chacras, huertas y sembradíos.

A fin de localizar vestigios de esta ocupación se prospectó el sector conocido actualmente como "Tapera Curruhuinca" en donde no se hallaron restos de estructuras pero sí material industrial asignable al siglo XX: tres fragmentos de porrones de gres; 11 fragmentos de vidrio (una base verde redonda con push up, cuatro fragmentos de cuerpos marrones -dos dejan ver inscripciones que se asocian a botellas de cerveza-, un fragmento de base marrón redonda, dos picos/cuellos marrones, tres botellas verdes de vino y/o sidra); dos elementos metálicos (una lata y un trozo de suncho); un fragmento de suela de goma con clavos y material de características indígenas: cinco desechos de talla y un guijarro pulido. Es importante mencionar que a muy pocos kilómetros de este lugar se encuentran las tumbas de Basilio Curruhuinca y su mujer.

La búsqueda de vestigios de la vida indígena en tiempos recientes condujo a su vez a los restos del Puesto Quintuman, cuyos datos también figuran en el Censo Indígena de 1927 (Familia Miranda-Quintoman) y en el que se realizó una prospección preliminar, proyectando un relevamiento sistemático y sondeos durante el próximo trabajo de campo. Hasta el momento se observaron en superficie, además de los desdibujados cimientos de la vivienda, los siguientes materiales: un fragmento de una base de frasco de vidrio, un fragmento de bisagra metálica, abundante material lítico (90 desechos de talla, 19 instrumentos, un núcleo) y un fragmento de cerámica indígena. Así mismo, se observaron restos de una estructura, posiblemente un "chenque" saqueado. Actualmente, un descendiente de la familia Quintuman (o Quintoman) es peón de una importante estancia ganadera del lugar y ya no posee tierras en la zona.

En suma, en las capas 0 y 1 y superficie de CP1, así como en los sitios asignables a indígenas recientes, se hallaron tanto artefactos confeccionados con técnicas indígenas como objetos de características industriales. Sin embargo, dado el carácter promediado de los contextos arqueológicos aquí presentados, la evidencia material recuperada hasta el momento no es suficiente para afirmar que se está ante situaciones de contacto.

\section{Síntesis y consideraciones finales}

El registro arqueológico recuperado en la excavación de la cueva principal de Casa de Piedra de Roselló (CP1) muestra una continuidad ininterrumpida de estratos que contienen restos de ocupaciones humanas desde aproximadamente los 9000 años AP hasta el siglo XX. Si bien las muestras fechadas hasta el momento no permiten tener cronologías lo suficientemente refinadas para poder estudiar períodos de corta duración ni para aseverar una continuidad ocupacional sin hiatos, el estudio de este sitio brinda valiosa información promediada sobre la vida de los diversos grupos humanos que la habitaron en el pasado. Aún restan los estudios del registro arqueofaunístico del sitio 
que podrían proporcionar indicadores de diferencias ocupacionales asociadas con el uso estacional de la cueva o con cambios ambientales a través del tiempo.

Tomando en cuenta las capas más tardías de CP1, se tiene hasta el momento un fechado de $c a .180$ años AP (de una estaca clavada) y otro de $c a$. 500 AP de la capa 1 que es en donde aparecen los primeros fragmentos de materiales industriales y restos faunísticos del período de contacto (oveja) conjuntamente con artefactos de confección indígena (especialmente material lítico), pero aún no se tiene un fechado para la capa 2 que sólo contiene materiales precontacto (Castro Esnal et al., 2016; Pérez de Micou et al., 2013). La capa 3 presenta cenizas del volcán Hudson del tipo H2, es decir de una antigüedad de ca. 4000 años cal. AP (C. Stern, comunicación personal, 2014). Esto muestra la necesidad de profundizar los estudios de las capas 1 y 2 , como acercamiento a los momentos inmediatamente previos y posteriores a la ocupación europeo/criolla de la cueva.

La historia reciente de las ocupaciones indígenas en la región, habla de familias que migraron desde el norte y que trajeron consigo un modo de vida agrícola pastoril (por ejemplo, los Curruhuinca y Quintuman) contrastante con el estilo de vida cazador recolector que se evidencia en los restos materiales indígenas de CP1. Las referencias de familias actuales con los antepasados más antiguos en la zona provienen de la colonia El Chalía (Castro Esnal, 2014; Castro Esnal et al., 2011; Pérez de Micou et al., 2009; Pinotti, 2001, 2004; Sacchi, 2012). Allí, se ha registrado la presencia de raspadores confeccionados sobre vidrio (Castro Esnal, 2014). Hasta el momento, en el área de Aldea Beleiro no se han encontrado este tipo de artefactos que, al menos para el caso de El Chalía, son evidencia material de una continuidad ocupacional y una incorporación de nuevos materiales europeo/criollos pero con la aplicación de técnicas tradicionales de talla. En este sentido las futuras investigaciones en Aldea Beleiro estarán dirigidas a dilucidar posibles continuidades y/o discontinuidades en los distintos momentos de su ocupación, a través de la profundización en el estudio de las capas superiores de $\mathrm{CP} 1$, como se mencionó anteriormente y del desarrollo de los trabajos en los espacios ocupados por indígenas en tiempos históricos y su comparación con los sitios en estudio de colonos europeo-criollos (Casanueva, 2016; Castro Esnal et al., 2016).

Se plantean como desafío los problemas metodológicos y de interpretación de las evidencias que conllevan abordar los espacios de contacto, especialmente en el caso de momentos recientes en los que no es tan clara la diferenciación de una ocupación de criollo-europeos de la de un asentamiento de indígenas con una economía agrícola pastoril. Se observan coincidencias entre las formas de asentarse, la producción e inclusive los objetos manipulados entre ambos grupos en tiempos históricos. Las distintas situaciones de contactos directos o indirectos produjeron variantes que dejaron improntas claras en algunas ocasiones y más desdibujadas en otras, el desafío consiste en poder identificar estos cambios. Se considera que la profundización de esta investigación permitirá un acercamiento a esta problemática.

\section{Agradecimientos}

A Cecilia Pérez de Micou, directora del Proyecto (financiado por la UBA y el CONICET); a Silvia García por su colaboración en la investigación antropológica; a Lucía Gutiérrez, Florencia Ronco, Esteban Alí Brouchoud, Cecilia Gutiérrez, Mariana Sacchi y María Luz Funes por su participación en las labores de campo y laboratorio; a Nora Kuperszmit por facilitarnos documentos históricos; a las Sras. Roselló por permitirnos el acceso e investigación en su campo; a las Familias Solsona y Pérez del Barrio por su gran hospitalidad y constante colaboración; a Narciso Quintuman; Diógenes Beleiro y a toda la Comuna de Aldea Beleiro. A los evaluadores cuyos comentarios contribuyeron a mejorar el manuscrito original. 


\section{Q Referencias citadas}

" Aguerre, A. M., Andrieu, J. M. e lantanos, N. (2017). Arqueología en Río Mayo, sudoeste del Chubut. Excavación en el Alero Dásovich: resultados preliminares. Intersecciones en Antropología, 18, 55-65.

》 Baeza, B. (2007). Los inspectores de tierra como productores identitarios de la frontera chileno-argentina en Patagonia Central. En Séptimo Congreso de Historia Social y Política de la Patagonia Argentino-Chilena, Historia, investigación e investigadores en y de la Patagonia. https://iesyppat.files.wordpress.com/2009/06/baezainspectores.doc (Acceso: 16 de febrero, 2015).

» Bandieri, S. (2005). Historia de la Patagonia. Buenos Aires: Editorial Sudamericana.

" Bate, L. F. (1979). Las investigaciones sobre los cazadores tempranos en Chile Austral. Trapananda, 1(2), 14-23.

"Casanueva, M. L. (2013). Colonos e Indígenas por Tierras Patagónicas. Una mirada arqueológica de la vida cotidiana transcurrida durante los siglos XVIII, XIXYXX. Saarbrücken: Publicia Editorial.

"Casanueva, M. L. (2016). Una visión arqueológica de la arquitectura doméstica en la Patagonia argentina de los siglos XVIII, XIX y XX. En F. Mena (Ed.), Arqueología de Patagonia: de mar a mar (pp. 69-79). Santiago de Chile: Ediciones CIEP y Ñire Negro Ediciones.

»Castro Esnal, A. (2014). Camino y Piedra. Rutas indígenas y Arqueología en la provincia de Chubut. Buenos Aires: Fundación de Historia Natural Félix de Azara.

»Castro Esnal, A., Casanueva, M. L., Sacchi, M. y Pérez de Micou, C. (2016). Estudios arqueológicos en Aldea Beleiro, SO del Chubut, Argentina. Nuevos fechados para el sitio Casa de Piedra. Revista del Museo de Antropología, 9(1), 7-12.

" Castro Esnal, A., Pérez de Micou, C. y Casanueva, M. L. (2017a). Early Holocene Occupation of the Forest-Steppe Ecotone of Southern South America: Evidence from Casa de Piedra de Roselló Cave (Chubut, Patagonia Argentina). PaleoAmerica, 3(3), 276-282.

"Castro Esnal, A., Sacchi, M. y Pérez de Micou, C. (2011). Aspectos generales de la tecnología lítica de los sitios de Colonia El Chalía (SO de la provincia de Chubut, Argentina). International Journal of South American Archaeology, 9, 28-40.

»Castro Esnal, A., Stern, C. y Pérez de Micou, C. (2017b). Aplicación de estudios geoquímicos sobre artefactos de obsidiana procedentes de contextos estratigráficos y superficiales en Aldea Beleiro, SO de Chubut (Patagonia, Argentina). Magallania, 45(1), 123-135.

"Censo de las Familias Indígenas de 1927. Inspección compuesta por las tribus de los Caciques Manuel Quilchamal y Basilio Curruhuinca y sus haciendas. Gobernación del Chubut. Expediente 345-2961. Rawson: Instituto Autárquico de Colonización y Fomento Rural de Chubut (IAC).

»Cirigliano, N. A. (2016). Movilidad de grupos indígenas y aprovechamiento de materias primas entre el extremo sur del macizo del Deseado y la cuenca del río Santa Cruz durante los últimos 2000 años (Provincia de Santa Cruz, Argentina). (Tesis Doctoral inédita), Universidad de Buenos Aires, Argentina.

"Dal Molin, C. (1998). Hoja Geológica 4572-IV Alto Río Senguer. SEGEMAR-Programa Nacional de Cartas Geológicas de la República Argentina 255. 
»Del Río, W. (2010). Del no-evento al genocidio. Eadem Utraque Europa. Revista de historia cultural e intelectual, 6(10/11), 219-254.

» Habegger, V. (2007). El mundo indígena frente a la dominación estatal. Norpatagonia, fines del siglo XIX - principios del siglo XX. Mundo Agrario, 8(15), http://www.scielo.org. ar/scielo.php?script=sci_arttext\&pid=S1515-59942007000200008\&lng=es\&nrm=iso (Acceso: 12 de julio, 2016).

» Levi, G. (1993). Sobre microhistoria. En P. Burke (Ed.), Formas de hacer Historia (pp. 119143). Madrid: Alianza Editorial.

» Maggiori, E. (2007). Aldea Beleiro. Historia de un pequeño pueblo de frontera. Rawson: Secretaría de Cultura de la Provincia de Chubut.

» Mena, F., Lucero, V., Reyes, O., Trejo, V. y Velázquez, H. (2000). Cazadores Tempranos y Tardíos en la Cueva Baño Nuevo-1, margen occidental de la estepa centropatagónica (XI Región de Aisen, Chile). Anales Instituto Patagonia, 28, 173-195.

» Mena, F. y Stafford, T. (2006). Contexto Estratigráfico y Fechación directa de esqueletos humanos del Holoceno Temprano en Cueva Baño Nuevo 1 (Patagonia Central, Chile). En J. Jiménez, S. González, J. Pompa y F. Ortiz (Eds.), Segundo Simposio Internacional del Hombre Temprano en América (pp. 139-54). Ciudad de México: INAH.

» Musso, L. M. (2015). Crónicas de dos familias pioneras y su entorno. Familias Beleiro y Musso. Comodoro Rivadavia: Vela al Viento Ediciones Patagónicas.

» Nuevo Delaunay, A., Goñi, R., Jiménez, N. L. y Cecuk, L. (2014). Marginalidad y adecuación en el siglo XX: dos casos de estudio en la cuenca del lago Strobel. En R. Goñi, J. B. Belardi, G. Cassiodoro y A. Re (Eds.), Arqueología de las cuencas de los lagos Cardiel y Strobel. Poblamiento humano y paleoambientes en Patagonia (pp. 187-198). Buenos Aires: Aspha.

"Pérez de Micou, C., Castro Esnal, A. y Sacchi, M. (2013). Estudios preliminares en el sitio Casa de Piedra, Estancia Roselló, sudoeste de Chubut. En A. F. Zangrando, R. Barberena, A. Gil, G. Neme, M. Giardina, L. Luna, C. Otaola, S. Paulides, L. Salgán y A. Tivoli (Eds.), Tendencias teórico-metodológicas y casos de estudio en la Arqueología de la Patagonia (pp. 213-218). San Rafael: Museo de Historia Natural de San Rafael.

»Pérez de Micou, C., Sacchi, M., Castro Esnal, A. y Funes, M. L. (2009). Estudios de Arqueología en la Colonia Indígena de Chalía. En F. Azar, E. M. Cúneo y S. N. Rodríguez (Eds.), Tras la senda de los ancestros: Arqueología de Patagonia (pp. 117-127). San Carlos de Bariloche: Universidad Nacional del Comahue.

» Pinotti, L. V. (2001). Sin embargo existimos. Reproducción biológica y cultural de una comunidad tehuelche. Buenos Aires: EUDEBA.

»Pinotti, L. V. (2004). Aquellos Tehuelches. Buenos Aires: Proyecto Editorial.

"Quijada, M. (2002). Repensando la frontera sur argentina: concepto, contenido, continuidades y discontinuidades de una realidad espacial y étnica (Siglos XVIII-XIX). Revista de Indias, LXII(224), 103-142.

» Ratto, S. (2001). El debate sobre la frontera a partir de Turner. La New Western History, los Borderlands y el estudio de las fronteras en Latinoamérica. Boletín del Instituto de Historia Argentina y Americana “Dr. Emilio Ravignani”, (Tercera Serie)24, $105-126$.

» Revel, J. (1995). Micro-análisis y construcción de lo social. Anuario del Instituto de Estudios Históricos y Sociales, 10, 125-143.

» Reyes O., Méndez, C., Mena, F. y Moraga, M. (2012). The bioanthropological evidence of a ca. 10,00o CALYBP ten-individual group in Central Patagonia. En L Miotti, M Salemme, N Flegenheimer y T. Goebel (Eds.), Southbound: Late Pleistocene Peopling of Latin America (pp. 167-171). Texas: Center for the Study of the First Americans. 
» Rock, J. (1981). Glass Bottles: Basic Identification. Yreka: Klamath National Forest, USDA.

»Sacchi, M. (2012). Materias Primas Líticas y Redes Sociales entre los grupos cazadoresrecolectores de Patagonia Centro-Meridional. (Tesis Doctoral inédita), Universidad de Buenos Aires, Argentina. 